\section{PRIMARY LEPTOMENINGEAL MEDULLOBLASTOMA: A RARE CLINICOPATHOLOGIC ENTITIY}

Daniel Hussey, Colleen D’Arcy

Anatomical Pathology Department, The Royal Children's

Hospital, Parkville, Vic, Australia

Medulloblastoma is a malignant embryonal neuroepithelial tumour accounting for $20 \%$ of childhood brain tumours. ${ }^{1}$ Presentation with primary leptomeningeal medulloblastoma without a dominant solid lesion is rare with only 6 previously reported cases. ${ }^{1}$ This case series reports $n=3$ further male paediatric patients with primary leptomeningeal medulloblastoma. All cases presented with symptoms of raised intracranial pressure and were initially treated for meningitis. Diagnosis was delayed on average 19 days secondary to equivocal radiology and CSF cytology. Dural biopsy confirmed the diagnosis and revealed anaplastic $(n=2)$ and classic $(n=1)$ medulloblastoma histology. Molecular subgrouping was consistent with non-WNT/non-SHH (group 3/4) medulloblastoma $(n=3)$. Post-treatment follow-up showed clinical and radiological remission in all cases. Our cases are unique as they are the only reported survivors of primary leptomeningeal medulloblastoma to date.

\section{Reference}

1. Guo X, Zhong D, Ma W. Primary leptomeningeal medulloblastoma: a rare case. Clin Neurol Neurosurg 2012; 114: $1181-4$.

\section{ADULT-ONSET SPINAL MUSCULAR ATROPHY WITH SMN DELETION AND ULTRASTRUCTURAL TUBULO- FILAMENTOUS INCLUSIONS IDENTICAL TO INCLUSION BODY MYOSITIS}

A. R. W. Kang ${ }^{1,2}$, M. Davis ${ }^{3}$, P. Lamont ${ }^{4}$, L. Griffiths ${ }^{5}$, J. Dyke $^{1,6}$, V. Fabian ${ }^{1}$

${ }^{1}$ PathWest Neuropathology, Royal Perth Hospital, Perth, WA, Australia; ${ }^{2}$ School of Medicine, University of Notre Dame, Fremantle, Perth, WA, Australia; ${ }^{3}$ PathWest Diagnostic Genomics, Nedlands, Perth, WA, Australia; ${ }^{4}$ Neurology and Neurogenetic Department, Royal Perth Hospital, Perth, WA, Australia; ${ }^{5}$ PathWest Electron Microscopic Unit, Nedlands, Perth, WA, Australia; and ${ }^{6}$ School of Medicine and Pharmacology, The University of Western Australia, Crawley, Perth, WA, Australia

Sporadic inclusion body myositis (IBM) is an inflammatory myopathy with the diagnostic criteria on electron microscopy of rimmed vacuoles and 15-18 $\mathrm{nm}$ intracytoplasmic/intranuclear tubulofilamentous inclusions. There is a spectrum of neuromuscular diseases with these features and to our knowledge, this is the first case of a genetically proven adult-onset spinal muscular atrophy illustrating inflammation, rimmed vacuoles and tubulofilamentous inclusions.

A 53-year-old male presented at 35 years with slowly progressive weakness, unsteady gait and atrophy of thighs and buttocks.
His upper limbs and distal legs were unaffected. Sensory examination was normal. There was no dysphagia, ocular disturbance, sphincteric involvement and no family history of a neuromuscular disorder. Serum CK was normal. The EMG and deltoid muscle biopsy showed a neurogenic process. Subsequent quadriceps muscle biopsy histology demonstrated denervation and focal myonecrosis, rimmed vacuoles and sarcoplasmic inclusions. Ultrastructurally, rimmed vacuoles with myelinoid phospholipid whorls and degenerate organelles, intracytoplasmic and intranuclear $16 \mathrm{~nm}$ tubular filaments were present. Single strand conformational polymorphism (SSCP) showed homozygous deletions of exons 7 and 8 of the SMN1 gene on chromosome $5 \mathrm{q}$

Our case of genetically proven adult-onset SMA confirms that rimmed vacuoles and $16 \mathrm{~nm}$.

IBM-like tubulofilamentous inclusions occur in a primary denervating condition.

References

1. Dubowitz V, Sewry C, Oldfors A. Muscle Biopsy: A Practical Approach. 5th ed. London: Elsevier, 2021.

2. Semino-Mora C, Dalakas MC. Rimmed vacuoles with betaamyloid and ubiquinated filamentous deposits in the muscles of patients with long-standing denervation (postpoliomyelitis muscular atrophy): similarities with inclusion body myositis. Hum Pathol 1998; 29: 1128-33.

\section{A DETAILED NEUROPATHOLOGICAL ASSESSMENT OF A WESTERN AUSTRALIAN CASE OF BALAMUTHIA MANDRILLARIS AMOEBIC ENCEPHALITIS}

Jason M. Dyke ${ }^{1,2}$, Jodi N. White ${ }^{3}$, David J. Speers ${ }^{2,4}$, Alexandra R. W. Kang ${ }^{1,5}$, Vicki A. Fabian


Australia; ${ }^{2}$ School of Medicine and Pharmacology, The University of Western Australia, Crawley, WA, Australia; ${ }^{3}$ PathWest Forensic Pathology, QEII Medical Centre, Nedlands, WA, Australia; ${ }^{4}$ PathWest Department of Microbiology, QEII Medical Centre, Nedlands, WA, Australia; and ${ }^{5}$ School of Medicine, University of Notre Dame, Fremantle, WA, Australia

Balamuthia mandrillaris is a protist, amphizoic protozoan that is free-living in the soil. This amoebae can rarely cause cutaneous disease and an amoebic encephalitis, the time-course of which ranges from subacute to fulminant. ${ }^{1}$

A 76-year-old male presented with a 1 week history of fevers and rigors, progressing rapidly to weakness with confusion and a GCS of 13 but without localising neurological signs. MRI brain and spine reported diffuse parenchymal and leptomeningeal disease, most marked within the median frontal lobes, favouring an infective/ haemorrhagic meningoencephalitis. No causative microorganism was identified on ante mortem investigation. Despite broad spectrum antimicrobial therapies, the patient deteriorated clinically. Brain death occurred 10 days after the initial presentation and the case was referred to the State Coroner's Office of WA. 